C-A/AP/\#196

March 2005

\title{
IR Optics Measurement Under Single Eigen Mode Activating
}

\author{
Yun Luo
}

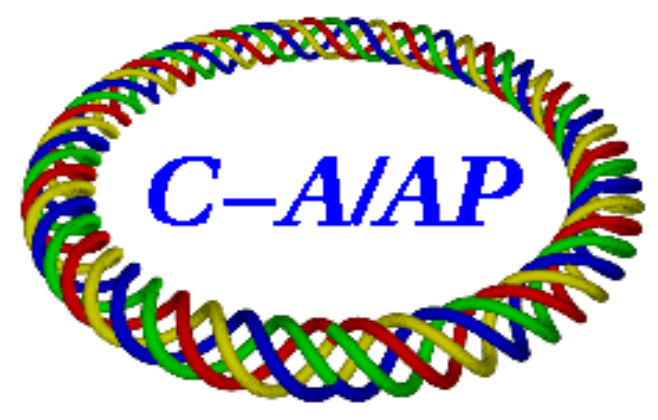

Collider-Accelerator Department

Brookhaven National Laboratory

Upton, NY 11973 
$\mathrm{CAD} / \mathrm{AP} / 196$

March 28, 2005

\title{
IR optics measurement under single eigen mode activating
}

\author{
Y. Luo \\ Brookhaven National Laboratory, Upton, NY 11973, USA
}

Linear coupling's action-angle parameterization is convenient for analytical calculation and turn-by-turn beam position monitor (BPM) data interpretation. In this article, the author demonstrates how to use it to extract the Twiss and coupling parameters in the interaction region (IR). The assumption is that there is one BPM in either side of the IR drift. The turn-by-turn BPM data sample were taken under the single eigen mode activating with the AC dipole at Relativistic Heavy Ion Collider (RHIC). Beside the full treatment, a fast estimate of the $\beta$ function in the IR center with the phase advance between the these two BPMs is given. The $\beta$ waist and the optics there also can be determined.

\section{Introduction}

The measurement of $\beta^{*}$ function at the colliding point plays an important role in the collider operation and development. The conventional method of $\beta^{*}$ measurement through knobing the first focusing quadrupole next to the interaction point (IP) doesn't provide high resolution. Another method to measure the second order tune shift due to the $\pm \Delta k$ changes to the first two focusing quadrupoles only applies to the symmetric IR optics collider. And its key parameter $\alpha_{\text {optics }}$ comes from the off-line optics simulation [1]. The two methods are based on the uncoupled optics assumption.

Linear coupling's action-angle paramterization is a strict and full treatment to the linearly coupled optics [2]. It is convenient for analytical calculation and turn-by-turn beam position monitor data interpretation. Since most colliders have beam position monitor (BPM) on either side of IP, the turn-by-turn $\left(x, x^{\prime}, y, y^{\prime}\right)$ data at these BPMs can be determined.

In this article, the author demonstrates how to use the action-angle parameterization to extract the full Twiss and coupling parameters in the interaction region (IR). The propagations of the Twiss and coupling parameters in the IR drift are also derived, from that the beta waist, where Twiss parameter $\alpha=0$, can be determined. And a method to fast check the $\beta$ function in the IR center only with the phase advance cross IR is introduced. These methods are applied to the BPM data example taken under the single eigen mode activating with the AC dipole at Relativistic Heavy Ion Collider (RHIC).

\section{Ingredients}

In this section we quickly review the linear coupling's action-angle parameterization and its application to the turn-by-turn BPM data interpretation under one eigen mode activating. And the propagations of the Twiss and coupling parameters in the IR drift is derived.

\subsection{Action-angle parameterization [2]}

For general two-dimensional linearly coupled motion, single-particle motion is

$$
\left(\begin{array}{c}
x \\
x^{\prime} \\
y \\
y^{\prime}
\end{array}\right)=\mathbf{P}\left(\begin{array}{c}
\sqrt{2 J_{I}} \cos \Phi_{I} \\
-\sqrt{2 J_{I}} \sin \Phi_{I} \\
\sqrt{2 J_{I I}} \cos \Phi_{I I} \\
-\sqrt{2 J_{I I}} \sin \Phi_{I I}
\end{array}\right),
$$

where $J_{I, I I}$ are the globally constant actions of the two eigen modes. $\Phi_{I, I I}$ are the eigen mode phases. One-turn phase advances for the two eigen modes are $2 \pi \mu_{I}, 2 \pi \mu_{I I}$, respectively. The $n$th turn's eigen pahses 
are

$$
\left\{\begin{array}{c}
\Phi_{I}=2 \pi \mu_{I}(n-1)+\phi_{I, 0} \\
\Phi_{I I}=2 \pi \mu_{I I}(n-1)+\phi_{I I, 0}
\end{array} .\right.
$$

$\phi_{I, 0}$ and $\phi_{I I, 0}$ are the eigen modes' initial phases, they are defined as

$$
\begin{gathered}
\left\{\begin{aligned}
\phi_{I, 0} & =\arctan \left(\frac{-S_{I}}{C_{I}}\right) \\
C_{I} & =\sum_{i=1}^{N} x_{i} \cos \left[2 \pi \mu_{I}(n-1)\right] \\
S_{I} & =\sum_{i=1}^{N} x_{i} \sin \left[2 \pi \mu_{I}(n-1)\right]
\end{aligned}\right. \\
\left\{\begin{aligned}
\phi_{I I, 0} & =\arctan \left(\frac{-S_{I I}}{C_{I I}}\right) \\
C_{I I} & =\sum_{i=1}^{N} y_{i} \cos [2 \pi \mu I I I(n-1)] \\
S_{I I} & =\sum_{i=1}^{N} y_{i} \sin \left[2 \pi \mu_{I I}(n-1)\right]
\end{aligned}\right.
\end{gathered}
$$

$N$ is the maximum turn for the calculation. According to eigen phase definitions, $p_{12}=p_{34}=0$,

$\mathbf{P}$ can be described in Twiss and coupling parameters,

$$
\mathbf{P}=\left(\begin{array}{cccc}
r \sqrt{\beta_{I}} & 0 & c_{11} \sqrt{\beta_{I I}}-\frac{c_{12} \alpha_{I I}}{\sqrt{\beta_{I I}}} & -\frac{c_{12}}{\sqrt{\beta_{I I}}} \\
-\frac{\alpha_{I} r}{\sqrt{\beta_{I}}} & \frac{r}{\sqrt{\beta_{I}}} & c_{21} \sqrt{\beta_{I I}}-\frac{c_{22} \alpha_{I I}}{\sqrt{\beta_{I I}}} & \frac{c_{22}}{\sqrt{\beta_{I I}}} \\
-\frac{c_{12} \alpha_{I}}{\sqrt{\beta_{I}}}-c_{22} \sqrt{\beta_{I}} & \frac{c_{12}}{\sqrt{\beta_{I}}} & r \sqrt{\beta_{I I}} & 0 \\
\frac{c_{11} \alpha_{I}}{\sqrt{\beta_{I}}}+c_{21} \sqrt{\beta_{I}} & -\frac{c_{11}}{\sqrt{\beta_{I}}} & -\frac{\alpha_{I I} r}{\sqrt{\beta_{I I}}} & \frac{r}{\sqrt{\beta_{I I}}}
\end{array}\right) .
$$

On the other hand, Twiss and coupling parameters can be obtained from $\mathbf{P}$,

$$
\begin{gathered}
\left\{\begin{aligned}
\beta_{I} & =\frac{p_{11}}{p_{22}} \\
\alpha_{I} & =\frac{p_{21}}{p_{22}} \\
\gamma_{I} & =\frac{1+\alpha_{I}^{2}}{\beta_{I}}=\frac{p_{21}^{2}+p_{22}^{2}}{p_{11} p_{22}}
\end{aligned}\right. \\
\left\{\begin{aligned}
\beta_{I I} & =\frac{p_{33}}{p_{44}} \\
\alpha_{I I} & =\frac{p_{43}}{p_{44}} \\
\gamma_{I I} & =\frac{1+\alpha_{I I}^{2}}{\beta_{I I}}=\frac{p_{43}^{2}+p_{44}^{2}}{p_{33} p_{44}} \\
r & =\sqrt{p_{11} p_{22}}=\sqrt{p_{33} p_{44}},
\end{aligned}\right.
\end{gathered}
$$

or

$$
\mathbf{C}=-\frac{1-r^{2}}{r} \mathbf{P}_{11} \mathbf{P}_{21}^{-1}
$$

\subsection{One eigen mode activating}

To measure the betatron optics, a single eigen mode motion is activated $[3,4,5]$. In the RHIC, AC dipoles are used for this purpose [6,7]. The strength modulation frequency of the AC dipole is close to one of the fractional eigen tunes. It has been shown that the beam emittance has no significant blow-up after the activating if the ramping of the $\mathrm{AC}$ dipole current is slow enough. The valid turn-by-turn BPM data are taken at the flattop of the AC dipole current.

For simplicity, Eq. (1) can be rewritten as

$$
\left(\begin{array}{c}
x \\
x^{\prime} \\
y \\
y^{\prime}
\end{array}\right)=\mathbf{F}\left(\begin{array}{c}
\cos \Phi_{I} \\
-\sin \Phi_{I} \\
\cos \Phi_{I I} \\
-\sin \Phi_{I I}
\end{array}\right)
$$




$$
\mathbf{F}=\left(\begin{array}{cccc}
p_{11} \sqrt{J_{I}} & 0 & p_{13} \sqrt{J_{I I}} & p_{14} \sqrt{J_{I I}} \\
p_{21} \sqrt{J_{I}} & p_{22} \sqrt{J_{I}} & p_{23} \sqrt{J_{I I}} & p_{24} \sqrt{J_{I I}} \\
p_{31} \sqrt{J_{I}} & p_{32} \sqrt{J_{I}} & p_{33} \sqrt{J_{I I}} & 0 \\
p_{41} \sqrt{J_{I}} & p_{42} \sqrt{J_{I}} & p_{43} \sqrt{J_{I I}} & p_{44} \sqrt{J_{I I}}
\end{array}\right)
$$

$\mathbf{F}$ includes the action information.

In this article, I assume that eigen mode I is more related to the horizontal plane than eigen mode II, and eigen mode II is more related to the vertical plane than eigen mode I. Thus, if only eigen mode I is activated, the elements in the last two column elements of $\mathbf{F}$ are zero, If only eigen mode II is activated, the elements in the first two column of $\mathbf{F}$ are zero.

$\mathbf{F}$ can be obtained from the turn-by-turn $\left(x, x^{\prime}, y, y^{\prime}\right)$ data at one point in the ring. For example, for the only eigen mode I activating motion,

$$
\begin{aligned}
& \left\{\begin{array}{l}
F_{11}=\frac{2}{N} \sum_{i=1}^{N} x_{i} \cos \left[2 \pi \mu_{I}(n-1)+\phi_{I, 0}\right] \\
F_{12}=-\frac{2}{N} \sum_{i=1}^{N} x_{i} \sin \left[2 \pi \mu_{I}(n-1)+\phi_{I, 0}\right] \\
F_{21}=\frac{2}{N} \sum_{i=1}^{N} x_{i}^{\prime} \cos \left[2 \pi \mu_{I}(n-1)+\phi_{I, 0}\right] \\
F_{22}=-\frac{2}{N} \sum_{i=1}^{N} x_{i}^{\prime} \sin \left[2 \pi \mu_{I}(n-1)+\phi_{I, 0}\right] \\
F_{31}=\frac{2}{N} \sum_{i=1}^{N} y_{i} \cos \left[2 \pi \mu_{I}(n-1)+\phi_{I, 0}\right] \\
F_{32}=-\frac{2}{N} \sum_{i=1}^{N} y_{i} \sin \left[2 \pi \mu_{I}(n-1)+\phi_{I, 0}\right] \\
F_{41}=\frac{2}{N} \sum_{i=1}^{N} y_{i}^{\prime} \cos \left[2 \pi \mu_{I}(n-1)+\phi_{I, 0}\right] \\
F_{42}=-\frac{2}{N} \sum_{i=1}^{N} y_{i}^{\prime} \sin \left[2 \pi \mu_{I}(n-1)+\phi_{I, 0}\right]
\end{array},\right. \\
& \left\{\begin{array}{l}
p_{i, 1}=\frac{F_{i, 1}}{\sqrt{J_{I}}}, \quad i=1,2,3,4 \\
p_{i, 2}=\frac{F_{i, 2}}{\sqrt{J_{I}}}, \quad i=1,2,3,4
\end{array} .\right.
\end{aligned}
$$

\subsection{Turn-by-turn $\left(x, x^{\prime}, y, y^{\prime}\right)$ data at DX/BPMs}

Here we dicuss how to obtain the turn-by-turn $\left(x, x^{\prime}, y, y^{\prime}\right)$ data in the IR region. In each interaction region of the RHIC, there are two dual-plane BPMs which are close to the IR separation magnet DX and facing to the interaction point, as shown in FIG. 1. We name these BPMs as DX/BPMs.

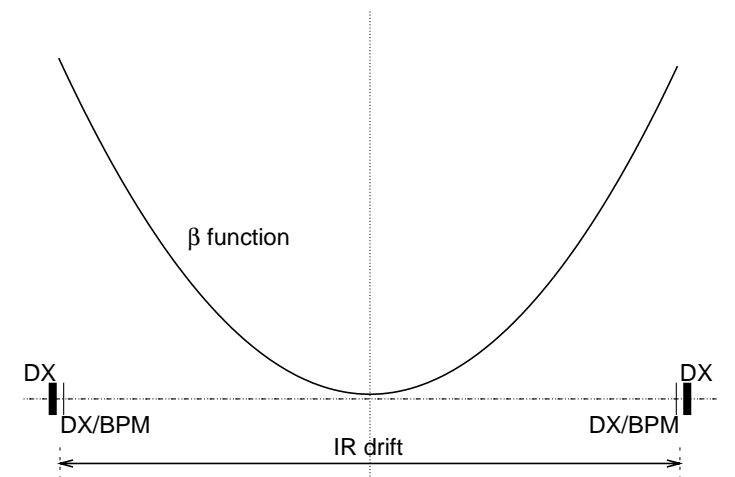

Figure 1: IR drift between the two DX/BPMs in one RHIC IR.

There is no other magnet between the two DX/BPMs if we ignore or switch off the detector magnets. Therefore, the turn-by-turn angles $\left(x^{\prime}, y^{\prime}\right)$ at the two DX/BPMs can be determined,

$$
\left\{\begin{array}{l}
x^{\prime}=\frac{x_{2}-x_{1}}{2 L} \\
y^{\prime}=\frac{y_{2}-y_{1}}{2 L}
\end{array} .\right.
$$


$2 L$ is the distance between the two DX/BPMs, $2 L=16.652 \mathrm{~m}$ for RHIC. $\left(x_{1}, y_{1}\right)$ are the BPM position readings at the up-stream DX/BPM, $\left(x_{2}, y_{2}\right)$ are the BPM position readings at the down-stream DX/BPM. The turn-by-turn angles $\left(x^{\prime}, y^{\prime}\right)$ are same across the IR drift.

\subsection{Optics parameter propagation in the IR drift}

Here we discuss the Twiss and coupling parameters' prorogations in the IR drift. It will be used for the $\beta$ waist determination and fast $\beta$ estimate at the center of IR.

At the $\beta$ waist where $\alpha_{I, I I}=0$,

$$
\mathbf{P}_{1}=\left(\begin{array}{cccc}
p_{11} & 0 & p_{13} & p_{14} \\
0 & p_{22} & p_{23} & p_{24} \\
p_{31} & p_{32} & p_{33} & 0 \\
p_{41} & p_{42} & 0 & p_{44}
\end{array}\right)
$$

For simplicity, we have assumed eigen mode I and eigen mode II's $\beta$ waists locate at the same point. If not, the following conclusions still hold. $\mathbf{T}_{1 \rightarrow 2}$ is the $4 \times 4$ drift transfer matrix from the waist to a point in the IR drift, say point 2 ,

$$
\mathbf{T}_{1 \rightarrow 2}=\left(\begin{array}{llll}
1 & l & 0 & 0 \\
0 & 1 & 0 & 0 \\
0 & 0 & 1 & l \\
0 & 0 & 0 & 1
\end{array}\right)
$$

$l$ is the distance from the waist to point 2. Matrix $\mathbf{G}$ defined by [2]'s Eq. (69) is

$$
\begin{aligned}
& \mathbf{G}=\mathbf{T}_{1 \rightarrow 2} \mathbf{P}_{1} \\
& =\left(\begin{array}{cccc}
p_{11} & l p_{22} & p_{13}+l p_{23} & p_{14}+l p_{24} \\
0 & p_{22} & p_{23} & p_{24} \\
p_{31}+l p_{41} & p_{32}+l p_{42} & p_{33} & l p_{44} \\
p_{41} & p_{42} & 0 & p_{44}
\end{array}\right) .
\end{aligned}
$$

According to Eqs. (78) and (79) in [2], and considering Eq.(4) at the waist, the Twiss parameters at point 2 can be determined with the above $\mathbf{G}$ Eq. (18),

$$
\left\{\begin{array}{l}
\widetilde{\beta}_{I}=\frac{p_{11}^{2}+l^{2} p_{22}}{p_{11} p_{22}}=\beta_{w, I}+\frac{l^{2}}{\beta_{w, I}} \\
\widetilde{\alpha}_{I}=-\frac{l p_{22}^{2}}{p_{11} p_{22}}=-\frac{l}{\beta_{w, I}}
\end{array}\right.
$$

similarily, for eigen mode II,

$$
\left\{\begin{array}{l}
\widetilde{\beta}_{I I}=\beta_{w, I I}+\frac{l^{2}}{\beta_{w, I I}} \\
\widetilde{\alpha}_{I I}=-\frac{l}{\beta_{w, I I}}
\end{array} .\right.
$$

The subscribe $w$ means the parameters are these at the $\beta$ waist.

Therefore, if knowing the Twiss parameters at point $2, \beta$ waist location and its $\beta$ value can be determined,

$$
\begin{aligned}
\left\{\begin{aligned}
\beta_{w, I} & =\frac{\widetilde{\beta}_{I}}{1+\widetilde{\alpha}_{I}^{2}} \\
\delta l_{w, I} & =-\widetilde{\alpha}_{I} \beta_{w, I}
\end{aligned}\right. \\
\left\{\begin{array}{l}
\beta_{w, I I}=\frac{\widetilde{\beta}_{I I}}{1+\widetilde{\alpha}_{I I}^{2}} \\
\delta l_{w, I I}=-\widetilde{\alpha}_{I I} \beta_{w, I I}
\end{array}\right.
\end{aligned}
$$

$\delta l_{w, I}$ and $\delta l_{w, I I}$ are eigen mode I and eigen mode II's waist's longitudinal locations with respect to point 2. 
According to Eqs. (70) and (72) in [2], the eigen mode phase advances from the $\beta$ waist to point 2 are given from $\mathbf{G}$ Eq. (18),

$$
\left\{\begin{array}{c}
\Delta \Phi_{I}=\tan ^{-1}\left(\frac{l p_{22}}{p_{11}}\right)=\tan ^{-1}\left(\frac{l}{\beta_{w, I}}\right) \\
\Delta \Phi_{I I}=\tan ^{-1}\left(\frac{l p_{34}}{p_{44}}\right)=\tan ^{-1}\left(\frac{l}{\beta_{w, I I}}\right)
\end{array} .\right.
$$

If we assume that the $\beta$ waist locates at the center of the two DX/BPMs, the total phase advances between them are

$$
\left\{\begin{array}{rl}
\Delta \Phi_{I} & =2 \tan ^{-1}\left(\frac{L}{\beta_{w, I}}\right) \\
\Delta \Phi_{I I} & =2 \tan ^{-1}\left(\frac{L}{\beta_{w, I I}}\right)
\end{array} .\right.
$$

$L$ is the distance from the IR center to the DX/BPM. Eq. (24) can be used for fast estimate of the $\beta_{c}$ at the IR center,

$$
\left\{\begin{array}{l}
\beta_{c, I}=\frac{L}{\tan \frac{\Delta \Phi_{I}}{2}} \\
\beta_{c, I I}=\frac{L}{\tan \frac{\Delta \Phi_{I I}}{2}}
\end{array} .\right.
$$

The merit of the fast estimate of $\beta_{c}$ with the DX/BPM phase advance is that it is insensitive to the BPM gains and offsets. And only the position turn-by-turn data are needed.

In the IR drift, knowing the coupling matrix $\mathbf{C}$ at one point in the IR drift, the coupling matrix $\widetilde{\mathbf{C}}_{w}$ at the waist can be obtained. Accroding Eq.(86) in [2], the coupling matrix $\mathbf{C}_{w}$ at the $\beta$ waist is

$$
\begin{aligned}
\mathbf{C}_{w} & =\mathbf{M}_{d} \mathbf{C M}_{d}^{-1}, \\
\mathbf{M}_{d} & =\left(\begin{array}{cc}
1 & \delta l_{w} \\
0 & 0
\end{array}\right),
\end{aligned}
$$

where $\delta l_{w}$ is the logintudinal drift length from point 1 to the waist.

\section{Measurements}

In the following, examples are given to demonstrate how to use the linear coupling's action-angle parameterization to extract the optics parameters in the IR. The BPM data example was taken from Horizontal AC dipole activating with eigen mode I's fractional tune frequency on Jan 10, 2005. The BPM data file name is Hacdipole-store-04.txt. For the vertical activating with the eigen mode II's fraction tune frequecy, the data processing procedure is similar to the eigen mode I activating situation.

To clarify the data processing procedure, we first calculate the Twiss and coupling parameters at the Blue ring IR6 center. The two dual-plane DX/BPMs are rbpm.b-g5 and rbpm.b-g6. Blue beam circulates from rbpm.b-g5 to rbpm.b-56. The turn-by-turn position data at the IR center are given by

$$
\left\{\begin{array}{l}
x_{c}=\frac{x_{1}+x_{2}}{2} \\
y_{c}=\frac{y_{1}+y_{2}}{2}
\end{array} .\right.
$$

The turn-by-turn angle data are obtained according to Eq. (15). FIG. 2 and FIG. 3 give the 2-D phase plots of $\left(x-x^{\prime}\right)$ and $\left(y-y^{\prime}\right)$ at the IR6 center, respectively.

\subsection{Matrix F}

With the above turn-by-turn $\left(x, x^{\prime}, y, y^{\prime}\right)$ data at Blue ring IR6 center, according to Eq. (11),

$$
\mathbf{F}=\left(\begin{array}{cccc}
1.2438 \times 10^{-5} & 6.5052 \times 10^{-19} & 0 & 0 \\
-1.0093 \times 10^{-6} & 1.2729 \times 10^{-5} & 0 & 0 \\
-6.4908 \times 10^{-7} & -9.7435 \times 10^{-7} & 0 & 0 \\
-4.5086 \times 10^{-7} & -9.2930 \times 10^{-7} & 0 & 0
\end{array}\right)
$$




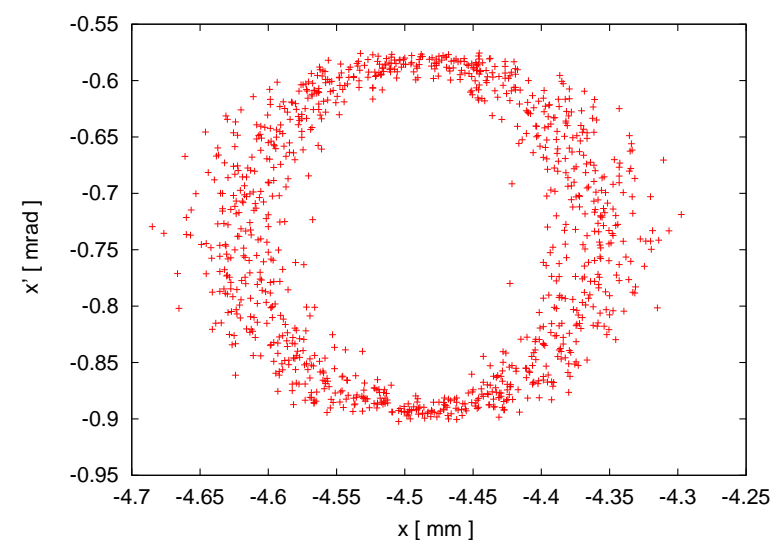

Figure 2: Turn-by-turn $\left(x, x^{\prime}\right)$ at IR6 center.

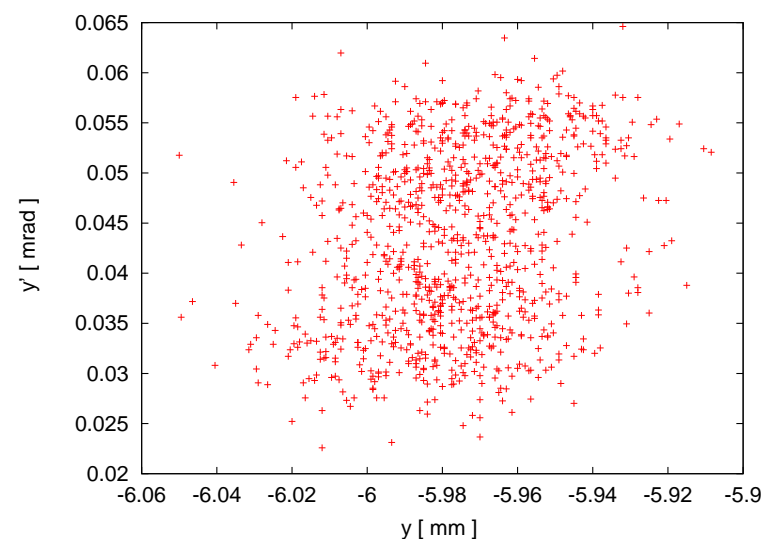

Figure 3: Turn-by-turn $\left(y, y^{\prime}\right)$ at IR6 center.

In this article, the SI unit is used.

Since the BPM data were taken from the horizontal AC dipole activating with eigne mode I's fractional tune frequency, so the right two column elements of matrix $\mathbf{F}$ are zero. The Twiss parameters related to eigen mode II can't be determined with only eigen mode I activating.

\section{$3.2 \quad r$ and $\sqrt{J_{I}}$}

According to Eq. (29),

$$
\left.r \sqrt{J_{I}}\right|_{I R 6}=\sqrt{F_{11} F_{22}}=1.2583 \times 10^{-5}\left[(\mathrm{~m} \cdot \mathrm{rad})^{-\frac{1}{2}}\right] .
$$

and according Eq. (10),

$$
\frac{\left\|\mathbf{F}_{21}\right\|}{\left\|\mathbf{F}_{11}\right\|}=\frac{\left\|\mathbf{P}_{21}\right\|}{\left\|\mathbf{P}_{11}\right\|}=\frac{1-r^{2}}{r^{2}}=0.001035 .
$$

Therefore, at the center of IR6,

$$
r=0.9995,
$$

and

$$
\sqrt{J_{I}}=1.2589 \times 10^{-5}\left[(\mathrm{~m} \cdot \mathrm{rad})^{-\frac{1}{2}}\right] .
$$

When $\mathrm{r}$ is close to 1 , it means the optics is well decoupled locally at that point. $\sqrt{J_{I}}$ is a global constant.

\subsection{Twiss and coupling parameters}

According Eq.(6), from Eq. (29), we get eigen mode I's Twiss parameter at the IR6 center as

$$
\left\{\begin{array}{l}
\beta_{c, I}=\frac{p_{11}}{p_{22}}=\frac{F_{11}}{F_{22}}=0.9771[\mathrm{~m}] \\
\alpha_{c, I}=-\frac{p_{21}}{p_{22}}=-\frac{F_{21}}{F_{22}}=0.0793
\end{array} .\right.
$$


Knowing $r, \sqrt{J_{I}}, \beta_{I}$, and $\alpha_{I}$, according to Eqs. (14) and (5), from Eq. (29), one gets the coupling matrix at the IR6 center as

$$
\mathbf{C}=\left(\begin{array}{cc}
0.0730 & -0.0765 \\
-0.0422 & 0.0584
\end{array}\right)
$$

\section{$3.4 \beta_{I}$ waist determination}

Knowing eigen mode I's Twiss parameters at the IR center, according to Eq. (21), the IR6 $\beta$ waist can be determined,

$$
\begin{gathered}
\beta_{w, I}=0.9710[\mathrm{~m}] \\
\delta l_{w, I}=-0.0770[\mathrm{~m}] .
\end{gathered}
$$

Negative $\delta l_{w, I}$ means the $\beta_{I}$ waist locates up stream with respect to the IR center.

According to Eq. (26), the coupling matrix $\mathbf{C}_{w}$ at the eigen mode I's $\beta$ waist can be calculated from that at the center,

$$
\mathbf{C}_{w}=\left(\begin{array}{cc}
0.0763 & -0.0752 \\
-0.0422 & 0.0551
\end{array}\right)
$$

\subsection{Summary of IR optics parameters}

Table 1 lists all IR centers' $\beta_{c, I}$ s from the phase advances between the two adjacent DX/BPMs, according to Eq. (25) . The phase advances between the relevant two DX/BPMs are also given.

Table 1: Fast estimate of IR center's $\beta_{c, I}$ s with the phase advances between DX/BPMs

\begin{tabular}{lcccccc} 
IRs & IR6 & IR8 & IR10 & IR12 & IR2 & IR4 \\
\hline$\Delta \Phi_{I}\left[{ }^{\circ}\right]$ & 166.695 & 166.474 & 147.064 & 141.788 & 150.637 & 119.395 \\
$\beta_{c, I}[\mathrm{~m}]$ & 0.9711 & 0.987 & 2.461 & 2.884 & 2.182 & 4.866
\end{tabular}

Table 2 lists the Twiss and coupling parameters at the IR centers from the full treatment with the action-angle parameterization. Since the rbpm.b-g2 BPM vertical data is not good, the coupling parameters are not available at IR2 center.

Table 2: Twiss and coupling parameters at the IR centers from the full treatment with action-angle paramterization

\begin{tabular}{lcccccc} 
IRs & $\beta_{c, I}$ & $\alpha_{c, I}$ & $c_{11}$ & $c_{12}$ & $c_{21}$ & $c_{22}$ \\
\hline IR6 & 0.9771 & 0.0793 & 0.0730 & -0.0765 & -0.0422 & 0.0584 \\
IR8 & 0.9885 & 0.0335 & 0.2549 & -1.7128 & -0.0455 & 1.0318 \\
IR10 & 2.4678 & 0.0536 & -0.0192 & -0.4945 & 0.0091 & -0.0213 \\
IR12 & 3.2762 & -0.3929 & 0.0484 & -1.2168 & 0.0337 & -0.2773 \\
IR2 & 2.5006 & 0.3972 & & & & \\
IR4 & 4.8918 & -0.0844 & -0.0854 & 0.03829 & -0.0344 & -0.2557
\end{tabular}

Comparing the $\beta_{c, I} \mathrm{~S}$ at the IR centers from Tables 1 and 2 , there is a big difference in the IR12. Looking into the turn-by-turn BPM data of the IR12 DX/BPMs, we found the down-stream BPM rbpm.b-g12 gave not good horizontal data.

Table 3 lists the $\beta_{w, I}$ waist loactions in the IRs and its $\beta_{w, I}$, coupling parameters there. $\delta l_{w, I}$ is the longitudinal offset with respect to the IR center.

Table 4 lists coupling parmater $r$ and eigen mode I's action $\sqrt{J_{I}}$ in IRs.

$\sqrt{J_{I}}$ is a global constant. The average of $\sqrt{J_{I}}$ in the IRs in Table 4 is

$$
\overline{\sqrt{J_{I}}}=1.18 \times 10^{-5}\left[(\mathrm{~m} \cdot \mathrm{rad})^{-\frac{1}{2}}\right] \text {. }
$$

It can be used to roughly extract the $r \sqrt{\beta_{I}}$ at the horizontal BPMs in the arcs,

$$
r \sqrt{\beta_{I}}=\frac{F_{11}}{\sqrt{J_{I}}}
$$


Table 3: Optics parameters at the $\beta_{I}$ waist in IRs.

\begin{tabular}{lcccccc} 
IRs & $\delta l_{w, I}[\mathrm{~m}]$ & $\beta_{w, I}$ & $c_{11}$ & $c_{12}$ & $c_{21}$ & $c_{22}$ \\
\hline IR6 & -0.0770 & 0.9710 & 0.0763 & -0.0752 & -0.0422 & 0.0551 \\
IR8 & -0.0331 & 0.9874 & 0.2812 & -1.9039 & -0.0455 & 1.0318 \\
IR10 & -0.1320 & 2.4607 & -0.0204 & -0.4955 & 0.0091 & -0.0213 \\
IR4 & 0.4100 & 4.8571 & -0.1007 & -0.0261 & -0.0348 & -0.2445
\end{tabular}

Table 4: $r$ and $\sqrt{J_{I}}$ in IRs.

\begin{tabular}{lcc} 
IRs & $r$ & $\sqrt{J_{I}}[\mathrm{~m} . \mathrm{mrad}]^{-\frac{1}{2}}$ \\
\hline IR6 & 0.999 & $1.26 \times 10^{-5}$ \\
IR8 & 0.911 & $1.17 \times 10^{-5}$ \\
IR10 & 0.997 & $1.11 \times 10^{-5}$ \\
IR4 & 0.988 & $1.09 \times 10^{-5}$
\end{tabular}

and $\frac{c_{12}}{r}$ at the dual-plane BPMs,

$$
\frac{c_{12}}{r}=\frac{F_{32}}{F_{11}}
$$

\section{Acknowledgments}

The author would like to thank M. Bai, R. Calaga, T. Satogata, and D. Trbojevic for their helps during this work. This work is support by the US DOE under contract No. DE-AC02-98CH10886.

\section{References}

[1] M.G. Minty, F. Zimmermann, Measurement and control of charged beams (Springer-Verlag), 2004

[2] Y. Luo, Phys. Rev. ST Accel. Beam 7, 124001 (2004).

[3] D. Sagan, Phys. Rev. ST Accel. Beam 4, 122801 (2001).

[4] D. Sagan et al., Phys. Rev. ST Accel. Beam 3, 102801 (2000).

[5] Y. Cai, Phys. Rev. E 68, 036501, (2003).

[6] M. Bai, et al., "Measuring beta function and phase advance in RHIC with an AC dipole", in proceedings of the 2003 Particle Accelerator Conference, Portland, p2204.

[7] R. Calaga, et al., "RHIC optics measurements at different working point", in proceedings of the 2004 European Particle Accelerator Conference, Lucerne, Switzerland, p1541. 